\title{
EEWMP: An IoT-Based Energy-Efficient Water Management Platform for Smart Irrigation
}

\author{
Rafi Ullah, ${ }^{1}$ Arbab Waseem Abbas, ${ }^{1}$ Mohib Ullah $\left(\mathbb{D}^{1},{ }^{1}\right.$ Rafi Ullah Khan ${ }^{(D)},{ }^{1}$ Irfan Ullah Khan, \\ Nida Aslam, ${ }^{2}$ and Sumayh S. Aljameel ${ }^{2}$ \\ ${ }^{1}$ Institute of Computer Science and Information Technology, The University of Agriculture, Peshawar, Pakistan \\ ${ }^{2}$ Department of Computer Science, College of Computer Science and Information Technology, \\ Imam Abdulrahman Bin Faisal University, Dammam, Saudi Arabia \\ Correspondence should be addressed to Rafi Ullah Khan; rafiyz@gmail.com
}

Received 24 January 2021; Revised 17 March 2021; Accepted 26 March 2021; Published 8 April 2021

Academic Editor: Shah Nazir

Copyright (c) 2021 Rafi Ullah et al. This is an open access article distributed under the Creative Commons Attribution License, which permits unrestricted use, distribution, and reproduction in any medium, provided the original work is properly cited.

Precision agriculture is now essential in today's world, especially for countries with limited water resources, fertile land, and enormous population. Smart irrigation systems can help countries efficiently utilize fresh water and use the excess water for barren lands. Smart water management platform (SWAMP) is an IoT-based smart irrigation project designed for efficient freshwater utilization in agriculture. The primary aim of SWAMP is to auto manage water reserves, distribution, and consumption of various levels, avoid over-irrigation and under-irrigation problems, and auto manage time to maximize production. This research proposed an energy-efficient water management platform (EEWMP), an improved version of SWAMP. EEWMP is an IoT-based smart irrigation system that uses field-deployed sensors, sinks, fusion centres, and open-source clouds. Both models' performance is evaluated in energy consumption, network stability period, packet sent to destination, and packet delivery ratio. The experimental results show that EEWMP consumes 30\% less energy and increases network stability twice than SWAMP. EEWMP can be used in different irrigation models such as drip irrigation, sprinkler irrigation, surface irrigation, and lateral move irrigation with subtle alterations. Moreover, it can also be used in small farms of third-world countries with their existing communication infrastructures such as $2 \mathrm{G}$ or $3 \mathrm{G}$.

\section{Introduction}

Water is an essential need for living things, and agriculture is the largest consumer of fresh water in the world with $70 \%$ consumption [1]. According to the researchers, the population growth has estimated to meet 10 billion in the twenty-first century. This rapid growth of population may create many demands and challenges for freshwater [2, 3]. Therefore, smart water management systems are essential to meet future demands of fresh water and food security. The irrigation system plays an essential role in crop yield as overirrigation and under-irrigation may significantly affect productivity and result in power and water wastage [4]. Precision irrigation, on the other hand, is an intelligent method that can be used to avoid the wastage of power and water while increasing productivity.
Internet of Things (IoT) is a system of physical objects (appliances with software and sensors, data centres, and machines) whose purpose is to collect and exchange data with each other over the Internet [5]. IoT's primary purpose is to increase machine-to-machine communication and take optimal decisions according to the situations but with less human interaction [6]. The invention and involvement of IoT revolutionized different processes are involved in many domains such as home security, equipment manufacturing, health monitoring, automated transportation, and especially agriculture [7]. In modern agriculture, the IoT is efficiently utilized in many subdomains such as precision farming [8], smart crop monitoring [9], soil quality [10], smart irrigation systems $[11,12]$, and many others.

For precision agriculture, a smart irrigation system is an essential requisite. The smart irrigation decision support 
system (SIDSS) is a novel technique that uses field deployed sensors to detect soil characteristics, weather and climate conditions, and crop conditions for irrigation [13]. In this regard, Kamienski et al. proposed an IoTbased smart water management platform (SWAMP) intelligent irrigation system $[1,14]$. SWAMP is one of the best available IoT-based irrigation systems that use SPARQL-based [15] semantic reasoning features and open-source cloud-based IoT platform FIWARE $[16,17]$. The SWAMP architecture comprises five different layers, including the device communication layer, acquisition security management layer, data management layer, water (irrigation) distribution layer, and water application services layer. In SWAMP, water management is further divided into three phases, i.e., reserve water, distribution, and consumption. Initially, SWAMP was implemented in Brazil and Europe at pilot project and produced promising results.

SWAMP is a big project and a combination of different technologies such as sensors, semantic computing, cloud services, communication protocols, drones, IoT, and many others $[1,14]$. However, many inherited issues are not addressed, which may affect the SWAMP project's efficiency. One of the main issues in the SWAMP project is related to sensors' energy consumptions as they send continuous/redundant reports. An energy-aware, efficient sensor communication model will increase the sensors' lifetime and reduce the project's cost.

This research aims to improve the SWAMP system's performance by proposing energy-efficient utilization techniques for different sensors (soil moisture, temperature, and water level measuring sensors). Therefore, in this research, we proposed an energy-efficient water management platform (EEWMP) with reduced redundant data strategies. The experimental results show that EEWMP consumes 30\% less energy and increases network stability twice than SWAMP. Similarly, due to increased network stability time, the destination's packets were 1.5 times more in EEWMP than SWAMP.

In Section 2, we introduce the SWAMP project briefly. In Section 3, we present a review of the latest literature on intelligent irrigation systems. In Sections 4 and 5, we discuss our proposed EEWMP model with its components and working. The methodology is discussed in Section 6. While in Sections 7 and 8, we discussed the results and conclusions with future work, respectively.

\section{SWAMP Project}

SWAMP is a collaborative project developed for intelligent irrigation and efficient freshwater utilization in agriculture $[1,14]$. The primary aim of SWAMP is to auto manage water reserves, distribution, and consumption of various levels, avoid over-irrigation and under-irrigation problems, and auto manage time to maximize production. The proposed SWAMP architecture is divided into five layers where each layer is dedicated to a specific responsibility, and each layer communicates with other layers using RDF [18], NGSI, or NGSI-LD [19] protocols.
The first layer of SWAMP architecture is called the device and communication layer, where different types of sensors are deployed in the field to acquire various types of information such as moisture and temperature. The information is collected from sensors using drones. The second layer is called data acquisition, security, and management layer, responsible for data acquisition and management. The third layer is called a data management layer responsible for data storage, processing, and distribution. This layer also uses semantic computing engines to process the data for the next layer. The fourth layer is called the water irrigation and distribution model layer, in which different types of traditional agriculture irrigation models are used to estimate the water need. The last layer of the SWAMP model is called the water application services layer, where the water is irrigated according to the need based on the data collected previously.

\section{Literature Review}

Channe et al. proposed an IoT-based multidisciplinary model for precision agriculture [20]. They proposed various applications of their model such as online agriculture data analysis, agricultural cloud, agribusiness, soil and weather analysis and predictions, and mobile app for farmers, vendors, and government representatives. They aimed to improve the crop production process with updated information about fertilizer utilization, soil analysis, and future need predations. In 2017, FIGARO (Flexible and precIse irriGation plAtform to improve faRm scale water prOductivity) project was started. The FIGARO project is a decision support system proposed to manage freshwater irrigation and improve production. They used different sensors, software, and cloud and involved field experts for optimal decisions [21]. Popovic et al. presented a case study on IoT-based precision agriculture platforms that use different sensors, IoT protocols, and analytic tools for ecology monitoring and precision agriculture [22]. Similarly, Kamilaris et al. proposed a SWAMP $[1,14]$ like a theoretical framework for IoT-enabled smart farming that facilitates farmers by providing accurate information based on semantic reasoning and real-time stream processing for decision-making [23].

Jaiganesh et al. proposed an IoT-based elegant farming model that uses mobile devices, information processing systems, and cloud services. They also proposed an agriculture cloud (Agro Cloud) module to collect, process, and store the data [24]. Li et al. proposed an IoT-based greenhouse management system that uses various android applications, sensors, communication protocols, and different hardware. Their proposed system offers general control functions such as temperature, humidity, and light adjustment functions. Furthermore, the system also offers various monitoring functions and weather forecasting functions [25]. Kiani and Seyyedabbasi proposed a sensor and IoTbased small farm monitoring system that monitors the temperature, humidity, and soil moisture to efficiently schedule the irrigation, harvesting, and cultivation plan [26]. Nurellari and Srivastava implemented an energy-efficient agriculture field monitoring system using IoT-enabled 
wireless sensors network. The system provides moisture, salinity, and soil temperature information to the farmers [27].

Karpagam et al. [28] proposed the IoT-enabled intelligent irrigation system for efficient water management and distribution. Their system monitors the water level in the field and supplies the water according to the need automatically with minimal human effort. Similarly, Gupta et al. [29] proposed an IoT-based intelligent irrigation system with a flood prevention system. They proposed a water level analysis system using well-maintained databases that measure the amount of rainfall and humidity level and predict future threats.

\section{Energy-Efficient Water Management Platform (EEWMP)}

The proposed energy-efficient water management platform (EEWMP) model is comprised of various sensors, computational devices, and services. The architecture of the proposed EEWMP is illustrated in Figure 1. The details of the proposed EEWMP system and architecture are discussed as follows.

4.1. Field Sensors. The sensor is a hardware device which is capable of collecting sensory information, processing it, and sending it to the base station using various communication technologies. In this research, we used three types of field sensors in the field, including soil moisture sensor, temperature sensor, and water level sensor, to collect the field data. This data of soil moisture, temperature, and the water level are then used in decision-making regarding irrigation. For experimentation, we used generic sensors available in Matlab.

4.2. In-Field Sink. The sink node's primary duty in wireless sensor network (WSN) is to collect the data from the sensors deployed in the vicinity using various strategies and conserve the nodes' energy by reducing communication traffic. In the EEEMP model, an in-field sink node is deployed to gather the whole field's data and send it to the outer sink. For experimentation, we used generic sensors available in Matlab.

4.3. Outer Sink and Fusion Centre. The outer sink node is used to collect the in-field sinks' data and then provide it to the embedded fusion centre. Fusion centre is a computational device precisely programmed for sharing information with the intellectual ability to remove redundant and empty data. The fusion centre's primary purpose is to reduce the communication traffic and conserve the energy consumed in various communication and computational tasks.

4.4. IoT Service Cloud. An IoT (Internet of Things) service cloud is an online service provided by different companies for different IoT-based services such as storage, processing, built-in and custom-built application to manage the data, and device management. Moreover, these clouds can also be accessible with desktops and handheld devices to get notifications and control devices. Several open-source and free IoT services are available such as FIWARE [30], Amazon, Microsoft, and Google's Cloud IoT [31]. In this research, the open-source cloud is used to reduce the cost. The provider cloud is used for registration/identification having a database used to save user data when the network is offline due to some reasons. The registration section is used to register the new client/end-user; identification is used to identify the end-user either the user has registered or not. All these devices of the provider cloud are connected to IoT connectivity.

4.5. Valves Controller. Valves controller is essential hardware used to control all the water values in the vicinity. Valves controller is IoT-enabled hardware connected with the IoT cloud to send the on/off signals to the small water channel valves according to the algorithm or user command.

4.6. Valve. Each farm is connected with the water canal through small water channels. Each small channel has an IoT-enabled valve used to control the water flow in the field when irrigation is needed. Each valve is connected with the valves controller.

4.7. User Connectivity. The user connectivity module is another important module of the whole proposed system. The user connectivity module is responsible for sending the notifications to the users regarding the farms' events and taking instructions from the users in response. The users can send and receive the information and commands using emails or cell phone applications.

\section{Working of EEWMP}

In the proposed energy-efficient water management platform (EEWMP) model, we introduced an in-field sink node whose duty is to collect the data from the sensors deployed in the field and thus save their energy. Moreover, the fusion centre can also reduce the communication traffic and can effectively conserve energy. In this section, we discuss the working of the EEWMP with the threshold value of different employed sensors.

Initially, all sensors are deployed in the 100-meter area with 10 meters distance and 10 to $15 \mathrm{~cm}$ depth with plant roots. One in-field sink node is deployed for each field to gather data from the deployed field sensors and send it to the outer sink. The outer sink node is embedded with fusion centre, responsible for filtering out the redundant and empty data and sending the clean data to the IoT service cloud. The IoT service cloud provides a device registration/identification process and application to manage and process the collected data. This application is also responsible for sending notifications to control the channel valves to auto start or stop the water if the irrigation is needed based on the collected data. 


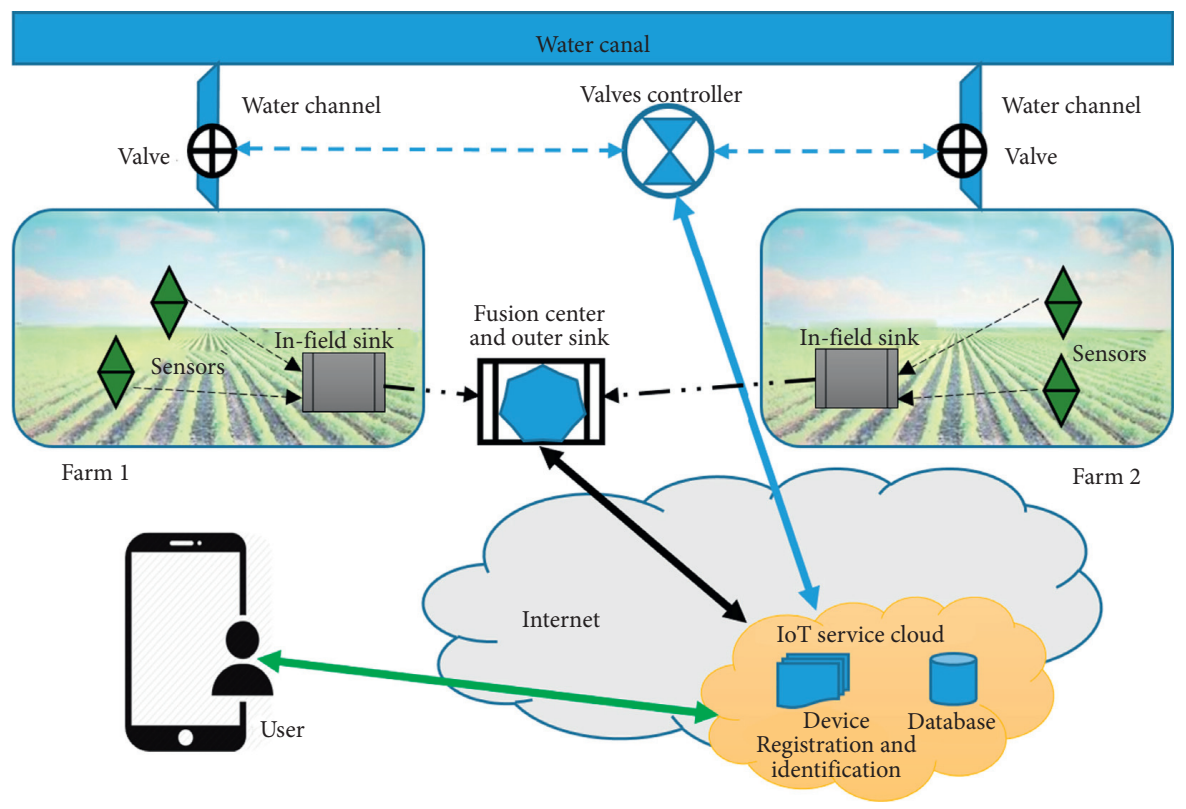

FIgURE 1: Architecture of proposed energy-efficient water management platform (EEWMP).

The threshold values of soil moisture for optimal field capacity are between $10 \%$ and $60 \%$. When soil moisture is greater than $10 \%$, the valves release water to the field until the moisture level reaches $60 \%$. The sensors will keep on the soil moisture level to the IoT cloud for further processing (Algorithm 1).

In the case of temperature sensors, there are three threshold values for three different temperature ranges. If the temperature is less than $15^{\circ} \mathrm{C}$, the water valve is programmed to release $3 \mathrm{~mm}$ to $4 \mathrm{~mm}$ water. If the temperature is between $15^{\circ} \mathrm{C}$ to $25^{\circ} \mathrm{C}$, the water valve is programmed to release $5 \mathrm{~mm}$ to $6 \mathrm{~mm}$ water. Moreover, if the temperature is more than $25^{\circ} \mathrm{C}$, the water valve is programmed to release $7 \mathrm{~mm}$ to $8 \mathrm{~mm}$ water (Algorithm 2).

The threshold of the water level sensor is based on two values: water level and tension test. Tension tests are used to check the soil moisture. If the water level is greater than or equal to $50 \%$ and the tension test value is greater than or equal to $20 \mathrm{cb}$ (Centibar), the measure to expel additional water is taken. When the water level is less than or equal to $40 \%$ and the tension test value is less than or equal to $50 \mathrm{cb}$, the valves are instructed to release water in the field (Algorithm 3).

\section{Methodology}

In this research, the proposed EEWMP model's performance is evaluated and compared with the SWAMP model. Both the systems are implemented and simulated in the MATLAB 2019a tool. We considered 100 nodes scenario deployed in $400 \times 400$ meters field and sink nodes at $200 \times 200$ meters for experimentation. The details of the nodes energies for different tasks and points are given in Table 1. Both models' performance is evaluated using energy consumption, network stability period, packet sent to destination, and packet delivery ratio.

\section{Results and Discussion}

In this research, we proposed an IoT-based smart and energy-efficient irrigation system, EEWMP. The proposed EEWMP system's performance is compared with the SWAMP model using simulation conducted in a MATLAB environment. The models' performance is evaluated in energy consumption, network stability period, packet sent to destination, and packet delivery ratio. In this section, we discuss the results of the experiments.

With the introduction of sink nodes in the proposed EEWMP model, the network energy consumption is effectively reduced compared with the SWAMP model. Due to the efficient utilization of the nodes' energy, the proposed EEWMP model's network lifetime also increased compared with the SWAMP model. According to the results, after 250 seconds, both EEWMP and SWAMP networks utilized $8 \%$ energy. However, at 500 seconds, SWAMP utilized 35\% energy whereas EEWMP utilized 30\% energy, which is 5\% less than SWAMP. Similarly, at 750 seconds, SWAMP utilized 69\% energy, whereas EEWMP utilized 50\% energy. At 1100 seconds, the SWAMP network is entirely exhausted, whereas EEWMP has more than $30 \%$ energy left at the same time. EEWMP network is completely exhausted at 2200 seconds, which is twice that of SWAMP. The energy consumption of the whole network in both models is illustrated in Table 2 and Figure 2.

Similarly, in network stability, the EEWMP model nodes are more stable than those in the SWAMP model. The results show that after 250 seconds, the number of alive nodes in the SWAMP model was 92, whereas the number of alive nodes in the EEWMP model was 93 . Similarly, at 500 seconds, the number of alive nodes was 65 and 70 in SWAMP and EEWMP models. At 1000 seconds, the number of alive nodes in SWAMP was nine, but at the same time, the number of alive nodes in EEWMP was 36. Table 3 illustrates 
Set time period $\mathbf{P}$

Initialize:

Monitor soil moisture SM (periodically $\mathbf{P}$ ) If $\mathrm{SM}<10 \%$

Open water valve for period $\mathbf{P}$

GOTO Initialize

If $\mathrm{SM}>60 \%$

Close water valve

GOTO Initialize

Algorithm 1: Algorithm for soil moisture.

Set time period $\mathbf{P}$

Initialize:

Monitor temperature temp (periodically $\mathbf{P}$ ) If Temp $<15^{\circ} \mathrm{C}$

Step 1: monitor water level WL ()

If $\mathrm{WL}<4 \mathrm{~mm}$

Open water valve for period $\mathbf{P}$

GOTO Step 1

If $\mathrm{WL}>4 \mathrm{~mm}$

Close water valve GOTO Initialize

If Temp $\geq 15^{\circ} \mathrm{C}$ and $\mathrm{Temp}<25^{\circ} \mathrm{C}$

Step 2: monitor water level WL ()

If $\mathrm{WL}<6 \mathrm{~mm}$

Open water valve for period $\mathbf{P}$ GOTO Step 2

If $\mathrm{WL}>6 \mathrm{~mm}$

Close water valve GOTO Initialize

If $\mathrm{Temp}>25^{\circ} \mathrm{C}$

Step 3: monitor water level WL ()

If $\mathrm{WL}<8 \mathrm{~mm}$

Open water valve for period $\mathbf{P}$ GOTO Step 3

If $\mathrm{WL}>8 \mathrm{~mm}$

Close water valve GOTO Initialize

Algorithm 2: Algorithm for soil temperature.

the number of alive nodes at different times in both models. The network stability period of both models is plotted in Figure 3.

In terms of the packet sent to the destination and packet delivery ratio, the EEWMP was more efficient and active than the SWAMP. According to the results (illustrated in Table 4), after 250 seconds, the total number of packets sent in the EEWMP scenario was 4900, whereas the total number of packets sent in the SWAMP scenario was 2500. The SWAMP model nodes were exhausted at 1100 seconds, and 
Set time period $\mathbf{P}$

Initialize:

Monitor soil moisture SM (periodically $\mathbf{P}$ )

Monitor tension test TT()

If $\mathrm{SM}<\mathbf{4 0 \%}$ and $\mathrm{TT}>\mathbf{5 0} \mathrm{cb}$ (Centibar)

Open water valve for period $\mathbf{P}$

GOTO Initialize

If $\mathrm{SM}>40 \%$ and $\mathrm{TT}>20 \mathrm{cb}$

Close water valve

GOTO Initialize

Algorithm 3: Algorithm for water level and tension test.

TABLE 1: Simulations parameters.

\begin{tabular}{lc}
\hline Parameters & Values \\
\hline Range of network & $400 \times 400 \mathrm{~m}^{2}$ \\
Location of sink & $200 \times 200 \mathrm{~m}^{2}$ \\
Number of nodes & 100 \\
Initial energy deployed to nodes & 0.5 joules \\
Transmission energy per node & $50\left(\mathrm{nano} \mathrm{jule)} \mathrm{nJ} / \mathrm{bit} / \mathrm{m}^{2}\right.$ \\
Receiver energy per node & $50 \mathrm{~nJ} / \mathrm{bit}^{2} / \mathrm{m}^{2}$ \\
Free space energy & $10 \mathrm{pico}-\mathrm{joule}(\mathrm{pJ})$ \\
Amplification energy & $0.0013 \mathrm{~nJ} / \mathrm{bit} / \mathrm{m}^{2}$ \\
Data aggregation energy & $5 \mathrm{pJ} / \mathrm{bit}$ \\
Maximum no. of rounds & 2500 \\
\hline
\end{tabular}

TABLE 2: Energy consumption of the whole scenario in percentage.

\begin{tabular}{|c|c|c|c|c|c|c|c|c|c|}
\hline \multirow{2}{*}{ Model } & \multicolumn{9}{|c|}{ Time (sec) } \\
\hline & 250 & 500 & 750 & 1000 & 1250 & 1500 & 1750 & 2000 & 2250 \\
\hline EEWMP (energy consumption in \%) & $8 \%$ & $30 \%$ & $50 \%$ & $64 \%$ & $70 \%$ & $79 \%$ & $92 \%$ & $97 \%$ & $100 \%$ \\
\hline SWAMP (energy consumption in \%) & $8 \%$ & $35 \%$ & $69 \%$ & $91 \%$ & $100 \%$ & $100 \%$ & $100 \%$ & $100 \%$ & $100 \%$ \\
\hline
\end{tabular}

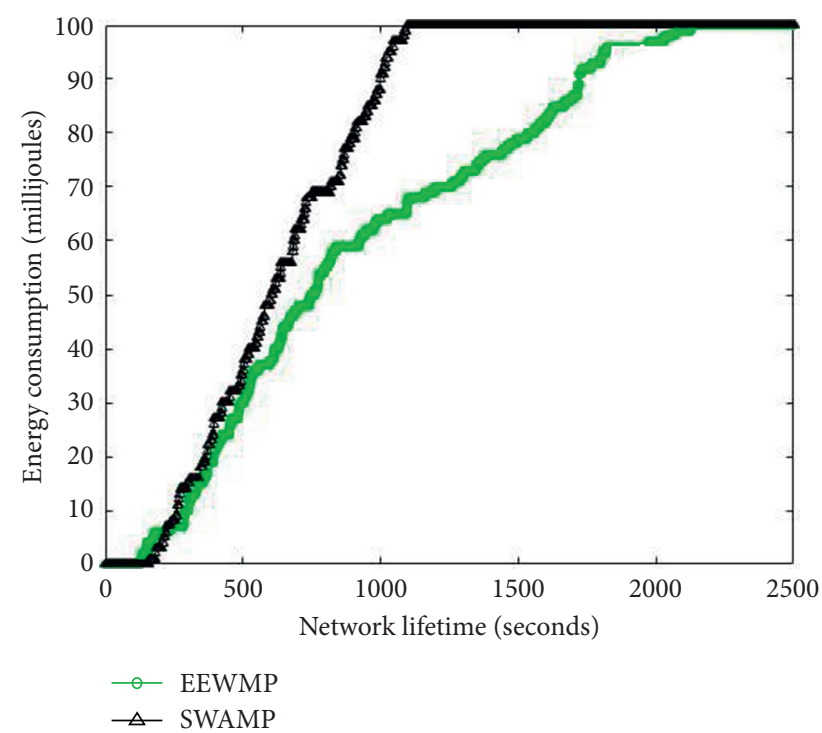

Figure 2: Energy consumption. at the end of the simulation (2500 seconds), the EEWMP model nodes sent 11500 more packets than the SWAMP model. Figure 4 shows the packets sent to the destination in both models.

Similarly, the packet delivery ratio in the EEWMP model was found much better than the SWAMP model due to the in-field sinks and fusion centre. In the initial stages of the simulation, the packet delivery ratio of SWAMP was slightly better than EEWMP; however, after 750 seconds, the packet delivery ratio of the EEWMP became better. The packet delivery ratio of both the models is illustrated in Table 5 and plotted in Figure 5.

Overall, the performance of the EEWMP is found better compared with the network model of SWAMP in terms of energy consumption, network stability period, packet sent to destination, and packet delivery ratio. The EEWMP model nodes consume a small amount of energy due to sink nodes and fusion centre, helping them survive more. Similarly, the extended lifetime of the node also increases the packet creation and delivery ratio. Furthermore, the network's life 
TABLE 3: Stability period.

\begin{tabular}{lcccccccc}
\hline Model & \multicolumn{9}{c}{ Time (sec) } \\
& 250 & 500 & 750 & 1000 & 1250 & 1500 & 1750 & 2000 \\
\hline EEWMP (alive nodes) & 93 & 70 & 50 & 36 & 30 & 21 & 8 & 3 \\
SWAMP (alive nodes) & 92 & 65 & 31 & 9 & 0 & 0 & 0 & 0 \\
\hline
\end{tabular}

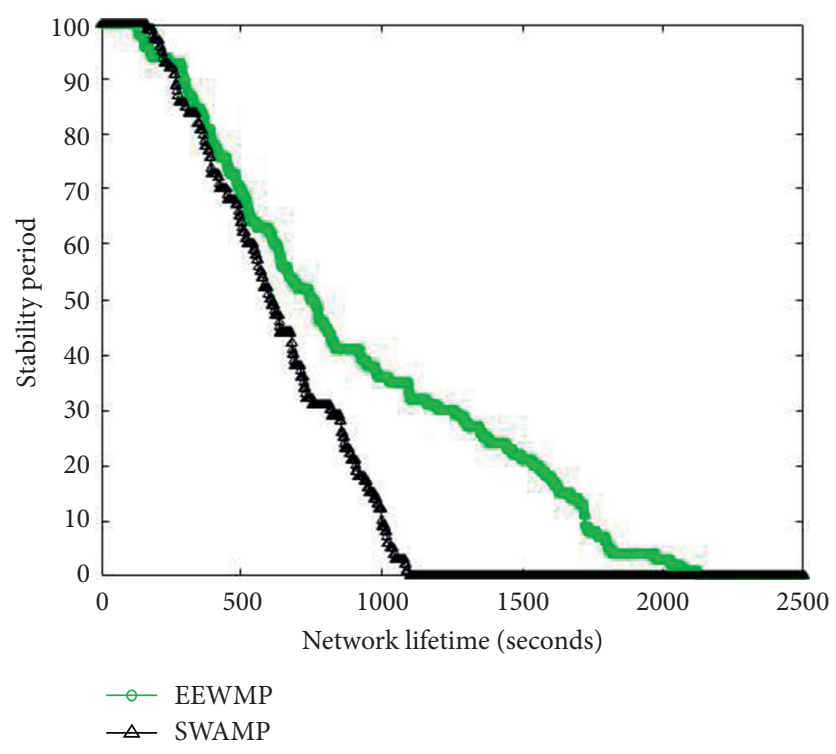

FIgURE 3: Stability period.

TABle 4: Packets sent to destinations.

\begin{tabular}{|c|c|c|c|c|c|c|c|c|c|}
\hline \multirow{2}{*}{ Model } & \multicolumn{9}{|c|}{ Time (sec) } \\
\hline & 250 & 500 & 750 & 1000 & 1250 & 1500 & 1750 & 2000 & 2250 \\
\hline EEWMP & 4900 & 9000 & 11900 & 14000 & 15600 & 17000 & 17900 & 18500 & 18500 \\
\hline SWAMP & 2500 & 4400 & 5700 & 6700 & 7000 & 7000 & 7000 & 7000 & 7000 \\
\hline
\end{tabular}

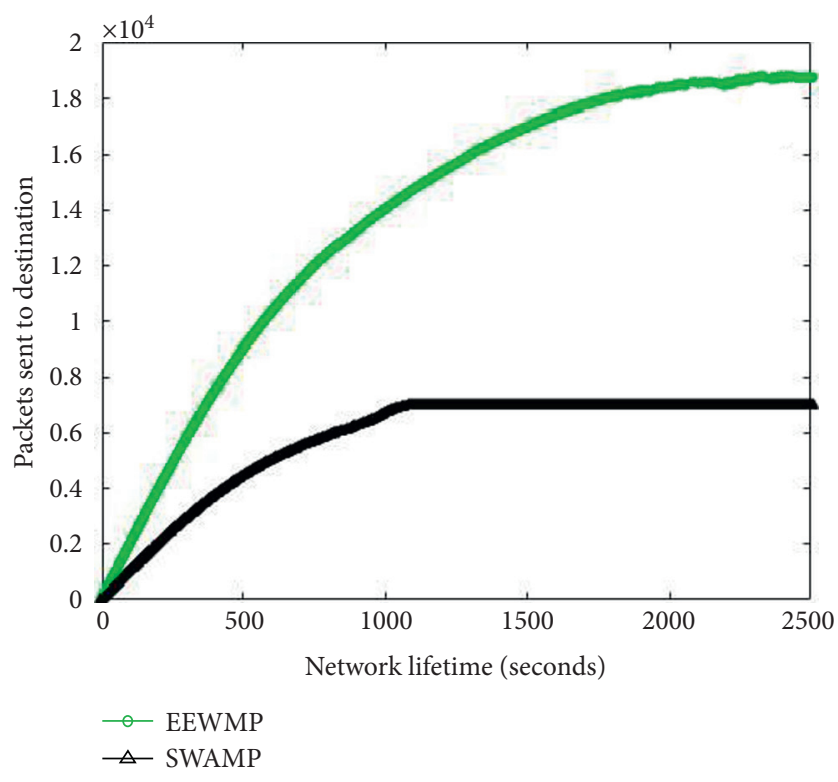

FIgURE 4: Packets sent to destinations. 
TABle 5: Packets delivery ratio.

\begin{tabular}{|c|c|c|c|c|c|c|c|c|c|}
\hline \multirow{2}{*}{ Model } & \multicolumn{9}{|c|}{ Time (sec) } \\
\hline & 250 & 500 & 750 & 1000 & 1250 & 1500 & 1750 & 2000 & 2250 \\
\hline EEWMP & 1.95 & 3.59 & 4.77 & 5.60 & 6.26 & 6.76 & 7.06 & 7.12 & 7.12 \\
\hline SWAMP & 2.22 & 3.97 & 5.03 & 5.51 & 5.53 & 5.53 & 5.53 & 5.53 & 5.53 \\
\hline
\end{tabular}

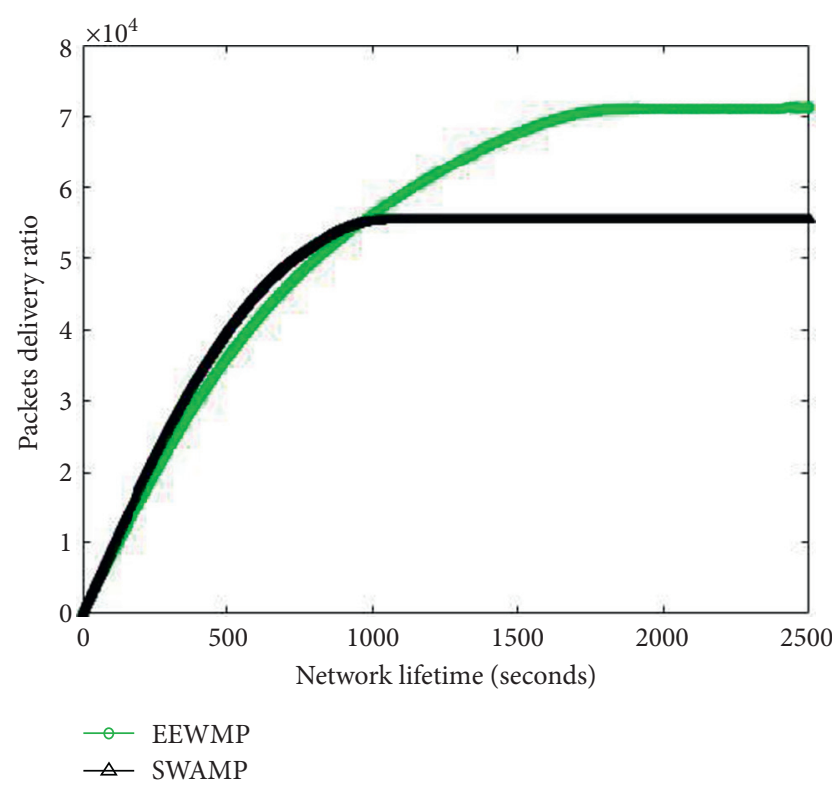

Figure 5: Packets delivery ratio.

can be further improved to find the optimized data sending frequency for nodes.

\section{Conclusions and Future Work}

The smart irrigation system is one of the essential needs for precision agriculture in the current time as we cannot afford fresh water wastage. IoT-based smart irrigation systems use field deployed sensors to detect soil characteristics, weather and climate conditions, and crop conditions for irrigation. SWAMP is a collaborative project developed for smart irrigation and efficient freshwater utilization in agriculture. The primary aim of SWAMP is to auto manage water reserves, distribution, and consumption of various levels, avoid over-irrigation and under-irrigation problems, and auto manage time to maximize production. This research improved the SWAMP network's performance by introducing an in-field sink and fusion centre use of open-source cloud to reduce costs. We called our improved model as energy-efficient water management platform (EEWMP). In the proposed EEWMP, the in-field sink node collects the data from the field's sensors and sends it to the fusion centre. The fusion centre aggregates the data and removes redundant information, thus reducing communication traffic and energy consumption. The results show that the performance of the EEWMP is found better compared with the network model of SWAMP in terms of energy consumption, network stability period, packet sent to destination, and packet delivery ratio. It was found that EEWMP consumes 30\% less energy and increases network stability time twice as compared with SWAMP. Similarly, due to increased network stability time, the destination's packets were 1.5 times more in EEWMP than SWAMP.

Based on the simulation results, it is safe to conclude that the introduction of sink nodes and fusion centre enhances the network performance in terms of data generation and energy consumption. EEWMP can be used in different irrigation models such as drip irrigation, sprinkler irrigation, surface irrigation, and lateral move irrigation with subtle alterations. Moreover, it can also be used in small farms of third-world countries with their existing communication infrastructures such as $2 \mathrm{G}$ or $3 \mathrm{G}$.

Precision agriculture is now essential in today's world, especially for countries with limited water resources, fertile land, and enormous population. Smart irrigation systems can help countries efficiently utilize fresh water and use the excess water for barren lands. In the future, we are interested in developing smart irrigation models for other irrigation systems, drip and sprinkler. We are also interested in utilizing other sensors to make smart irrigation models and algorithms for different soil types such as gravel, silt, loam, sand, and barren land.

\section{Data Availability}

It is a simulation-based research, and no data were used.

\section{Conflicts of Interest}

The authors declare that they have no conflicts of interest regarding the publication of this paper.

\section{References}

[1] C. Kamienski, J.-P. Soininen, M. Taumberger et al., "Smart water management platform: iot-based precision irrigation for agriculture," Sensors, vol. 19, no. 2, p. 276, 2019.

[2] F. Aquastat, "Water uses," www. fao. org/nr/water/aquastat/ water_use/index. stm, 2016.

[3] M. Rodell, J. S. Famiglietti, D. N. Wiese et al., "Emerging trends in global freshwater availability," Nature, vol. 557, no. 7707, pp. 651-659, 2018.

[4] R. G. Perea, A. Daccache, J. R. Díaz, E. C. Poyato, and J. W. Knox, "Modelling impacts of precision irrigation on crop yield and in-field water management," Precision Agriculture, vol. 19, pp. 497-512, 2018.

[5] P. P. Ray, "A survey on Internet of Things architectures," Journal of King Saud University - Computer and Information Sciences, vol. 30, no. 3, pp. 291-319, 2018.

[6] E. G. Popkova, E. N. Egorova, E. Popova, and U. A. Pozdnyakova, "The model of state management of economy on the basis of the internet of things," in Ubiquitous Computing and the Internet of Things: Prerequisites for the 
Development of ICT, pp. 1137-1144, Springer, New York, NY, USA, 2019.

[7] P. Asghari, A. M. Rahmani, and H. H. S. Javadi, "Internet of Things applications: a systematic review," Computer Networks, vol. 148, pp. 241-261, 2019.

[8] R. Finger, S. M. Swinton, N. El Benni, and A. Walter, Precision Farming at the Nexus of Agricultural Production and the Environment, Annual Review of Resource Economics, vol. 11, no. 1, pp. 313-335, 2019.

[9] D. Popescu, L. Ichim, F. Stoican, and C. Dragana, "Hierarchical processing of signals for smart crop monitoring," in Proceedings of the 2019 8th International Conference on Systems and Control (ICSC), pp. 265-270, Marrakech, Morocco, 2019.

[10] M. A. Ali, L. Dong, J. Dhau, A. Khosla, and A. Kaushik, "Perspective-electrochemical sensors for soil quality assessment," Journal of The Electrochemical Society, vol. 167, Article ID 037550, 2020.

[11] L. García, L. Parra, J. M. Jimenez, J. Lloret, and P. Lorenz, "IoT-based smart irrigation systems: an overview on the recent trends on sensors and IoT systems for irrigation in precision agriculture," Sensors, vol. 20, no. 4, p. 1042, 2020.

[12] S. Chaudhry and S. Garg, "Smart irrigation techniques for water resource management," in Smart Farming Technologies for Sustainable Agricultural Development, pp. 196-219, IGI Global, Philadelphia, PA, USA, 2019.

[13] A. Srilakshmi, J. Rakkini, K. Sekar, and R. Manikandan, "A comparative study on Internet of Things (IoT) and its applications in smart agriculture," Pharmacognosy Journal, vol. 10, 2018.

[14] C. Kamienski, J.-P. Soininen, and M. Taumberger, "Swamp: an IoT-based smart water management platform for precision irrigation in agriculture," in Global Internet of Things Summit, IEEE, New York, NY, USA, 2018.

[15] A. Fiorentino, J. Zangari, and M. Manna, "DaRLing: a Datalog rewriter for OWL 2 RL ontological reasoning under SPARQL queries," Theory and Practice of Logic Programming, vol. 20, no. 6, pp. 958-973, 2020.

[16] V. Araujo, K. Mitra, S. Saguna, and C. Åhlund, "Performance evaluation of FIWARE: a cloud-based IoT platform for smart cities," Journal of Parallel and Distributed Computing, vol. 132, pp. 250-261, 2019.

[17] M. A. Rodriguez, L. Cuenca, and A. Ortiz, "FIWARE open source standard platform in smart farming-a review," in Proceedings of the Working Conference on Virtual Enterprises, pp. 581-589, Cardiff, UK, 2018.

[18] M. Wylot, M. Hauswirth, P. Cudré-Mauroux, and S. Sakr, "RDF data storage and query processing schemes," ACM Computing Surveys, vol. 51, no. 4, pp. 1-36, 2018.

[19] F. Viola, F. Antoniazzi, C. Aguzzi, C. Kamienski, and L. Roffia, "Mapping the NGSI-LD context model on top of a SPARQL event processing architecture: implementation guidelines," in Proceedings of the 2019 24th Conference of Open Innovations Association (FRUCT), pp. 493-501, Moscow, Russia, 2019.

[20] H. Channe, S. Kothari, and D. Kadam, "Multidisciplinary model for smart agriculture using internet-of-things (IoT), sensors, cloud-computing, mobile-computing \& big-data analysis," International Journal Computer Technology \& Applications, vol. 6, pp. 374-382, 2015.

[21] L. Doron, "Flexible and precise irrigation platform to improve farm scale water productivity," Impact, vol. 2017, no. 1, pp. 77-79, 2017.

[22] T. Popović, N. Latinović, A. Pešić, Ž. Zečević, B. Krstajić, and S. Djukanović, "Architecting an IoT-enabled platform for precision agriculture and ecological monitoring: a case study," Computers and Electronics in Agriculture, vol. 140, pp. 255265, 2017.

[23] A. Kamilaris, F. Gao, F. X. Prenafeta-Boldu, and M. I. Ali, "Agri-IoT: a semantic framework for Internet of Thingsenabled smart farming applications," in Proceedings of the 2016 IEEE 3rd World Forum on Internet of Things (WF-IoT), pp. 442-447, Reston, VA, USA, 2016.

[24] S. Jaiganesh, K. Gunaseelan, and V. Ellappan, "IOT agriculture to improve food and farming technology," in Proceedings of the 2017 Conference on Emerging Devices and Smart Systems (ICEDSS), pp. 260-266, Piscataway, NJ, USA, 2017.

[25] Z. Li, J. Wang, R. Higgs, L. Zhou, and W. Yuan, "Design of an intelligent management system for agricultural greenhouses based on the internet of things," in Proceedings of the 2017 IEEE International Conference on Computational Science and Engineering (CSE) and IEEE International Conference on Embedded and Ubiquitous Computing (EUC), pp. 154-160, Piscataway, NJ, USA, 2017.

[26] F. Kiani and A. Seyyedabbasi, "Wireless sensor network and internet of things in precision agriculture," International Journal of Advanced Computer Science and Applications (Ijacsa), vol. 9, 2018.

[27] E. Nurellari and S. Srivastava, "A practical implementation of an agriculture field monitoring using wireless sensor networks and IoT enabled," in Proceedings of the 2018 IEEE International Symposium on Smart Electronic Systems (iSES)(Formerly iNiS), pp. 134-139, Hyderabad, India, 2018.

[28] J. Karpagam, I. I. Merlin, P. Bavithra, and J. Kousalya, "Smart irrigation system using IoT," in Proceedings of the 2020 6th International Conference on Advanced Computing and Communication Systems (ICACCS), pp. 1292-1295, Coimbatore, India, 2020.

[29] S. Gupta, V. Malhotra, and V. Vashisht, "Water irrigation and flood prevention using IOT," in Proceedings of the 2020 10th International Conference on Cloud Computing, pp. 260-265, Data Science \& Engineering, Coimbatore, India, 2020.

[30] M. A. Rodriguez, L. Cuenca, and A. Ortiz, "FIWARE open source standard platform in smart farming-a review," in Working Conference on Virtual Enterprises, pp. 581-589, Springer, Cham, Switzerland, 2018.

[31] P. Pierleoni, R. Concetti, A. Belli, and L. Palma, "Amazon, google and microsoft solutions for iot: architectures and a performance comparison," IEEE Access, vol. 8, pp. 5455-5470, 2019. 